\title{
Simulations with current constraints of ELM-induced tungsten melt motion in ASDEX Upgrade
}

\author{
E. Thorén ${ }^{1}$, B. Bazylev ${ }^{2}$, S. Ratynskaia ${ }^{1}$, P. Tolias ${ }^{1}$, K. \\ Krieger $^{3}$, R. A. Pitts ${ }^{4}$, S. Pestchanyi ${ }^{2}$, M. Komm ${ }^{5}$, B. Sieglin ${ }^{3}$, \\ the EUROfusion MST1 Team $\ddagger$ and the ASDEX Upgrade Team ${ }^{3}$ \\ ${ }^{1}$ KTH Royal Institute of Technology, SE-10044 Stockholm, Sweden \\ ${ }^{2}$ Karlsruhe Institute of Technology, P.O. Box 3640, D-76021 Karlsruhe, Germany \\ ${ }^{3}$ Max-Planck-Institut für Plasmaphysik, 85748 Garching b. München, Germany \\ ${ }^{4}$ ITER Organization, Route de Vinon-sur-Verdon, CS 90 046, 13067 St. Paul Lez \\ Durance, France \\ ${ }^{5}$ Institute of Plasma Physics CAS, Za Slovankou 3, 18200 Prague 8, Czech Republic \\ E-mail: emitho@kth.se
}

\begin{abstract}
Melt motion simulations of recent ASDEX Upgrade experiments on transient-induced melting of a tungsten leading edge during ELMing H-mode are performed with the incompressible fluid dynamics code MEMOS 3D. The total current flowing through the sample was measured in these experiments providing an important constraint for the simulations since thermionic emission is considered to be responsible for the replacement current driving melt motion. To allow for a reliable comparison, the description of the space-charge limited regime of thermionic emission has been updated in the code. The effect of non-periodic aspects of the spatio-temporal heat flux in the temperature distribution and melt characteristics as well as the importance of current limitation are investigated. The results are compared with measurements of the total current and melt profile.
\end{abstract}

\section{Introduction}

Unsuccessfully mitigated edge localized modes (ELMs) can cause transient tungsten (W) melting, which is a primary concern for ITER $[1,2]$. The macroscopic motion of the $\mathrm{W}$ melt driven by electromagnetic forces or pressure gradients can lead to large-scale erosion compromising the power handling capabilities of the local plasma-facing components (PFCs), whereas the rapid growth of hydrodynamic instabilities can lead to droplet ejection compromising plasma performance. In addition to potential lifetime reduction, the accumulation of eroded material can also cause bridging of the gaps between adjacent

‡ See author list of "H. Meyer et al. 2017 Nucl. Fusion 57 102014" 
W monoblocks possibly increasing eddy current forces [1-3]. Consequently, dedicated experiments have been designed in contemporary devices against which simulation tools being used to assess the consequences of melting on ITER can be tested. Simulations of melt motion are generally based on coupling the Navier-Stokes equations for the liquid metal with the heat conduction equation and supplementing the system with appropriate boundary conditions that are dictated by the incident plasma and the thermo-electrical material connections. In particular, the MEMOS 3D melt motion code solves the incompressible fluid equations within the shallow water approximation [4].

This work is focused on MEMOS 3D simulations [4] of recent ASDEX Upgrade (AUG) experiments on repetitive transient $\mathrm{W}$ melting during ELMing H-mode [5]. A W sample was exposed at the outer divertor target to Type 1 ELMing H-mode discharges producing transient parallel power flux densities of $\sim 1 \mathrm{GW} / \mathrm{m}^{2}$ over ms timescales at a frequency of ca $70 \mathrm{~Hz}$. The sample design is very similar to that employed in previous JET experiments [6] and provides a leading edge of $1 \mathrm{~mm}$ height nearly perpendicular to the magnetic field lines arriving at glancing angle to the target surface. The ELMinduced power flux densities are similar to those targeted in mitigated ITER ELMs and the order-of-magnitude difference in the particle temperatures (and thus the plasma pressures) between the devices should not lead to large differences in the melt layer dynamics for the specific exposure configuration. A key enhancement with respect to the JET experiment is the electrical isolation of the sample and hence a capability to measure the total current flowing through the sample, providing an important constraint for the simulations. However, unlike in the JET experiment, no direct top surface IR measurements of the loaded sample are available for these AUG exposures.

Following a brief presentation of the experimental conditions and some updates of the MEMOS 3D code mainly concerning the modelling of space-charge limited thermionic emission $[7,8]$, we present a comparison between the total measured current and the computed thermionic current both for coherently averaged and raw (corrected for IR artifacts) ELM plasma heat flux profiles. The effect of non-periodic aspects of the spatio-temporal ELM heat flux as well as the role of space-charge limitation are investigated. Melt characteristics such as the poloidal extent, pool depth and build-up are calculated for the two heat load scenarios and compared with the experiment.

\section{Experimental background and input}

The AUG experiments on transient $\mathrm{W}$ melting are described in detail in Ref.[5]. The experimental set-up and the exposure geometry are illustrated in Fig.1. Both leading edge and sloped lamellae were exposed for a gradually increased duration in order to avoid the occurrence of bulk melting (namely melting also in-between ELMs). The pulses relevant for the transient melting of the leading edge are \#33504, \#33508, \#33509 ELMing H-mode shots, very similar in terms of input power and ELM characteristics, with $B_{\mathrm{t}}=-2.5 \mathrm{~T}, I_{\mathrm{p}}=0.8 \mathrm{MA}, P=7.5 \mathrm{MW}(\mathrm{NBI}), 2 \mathrm{MW}(\mathrm{ECRH})$. In this paper, we shall focus exclusively on \#33504. The input necessary for modelling was constructed 

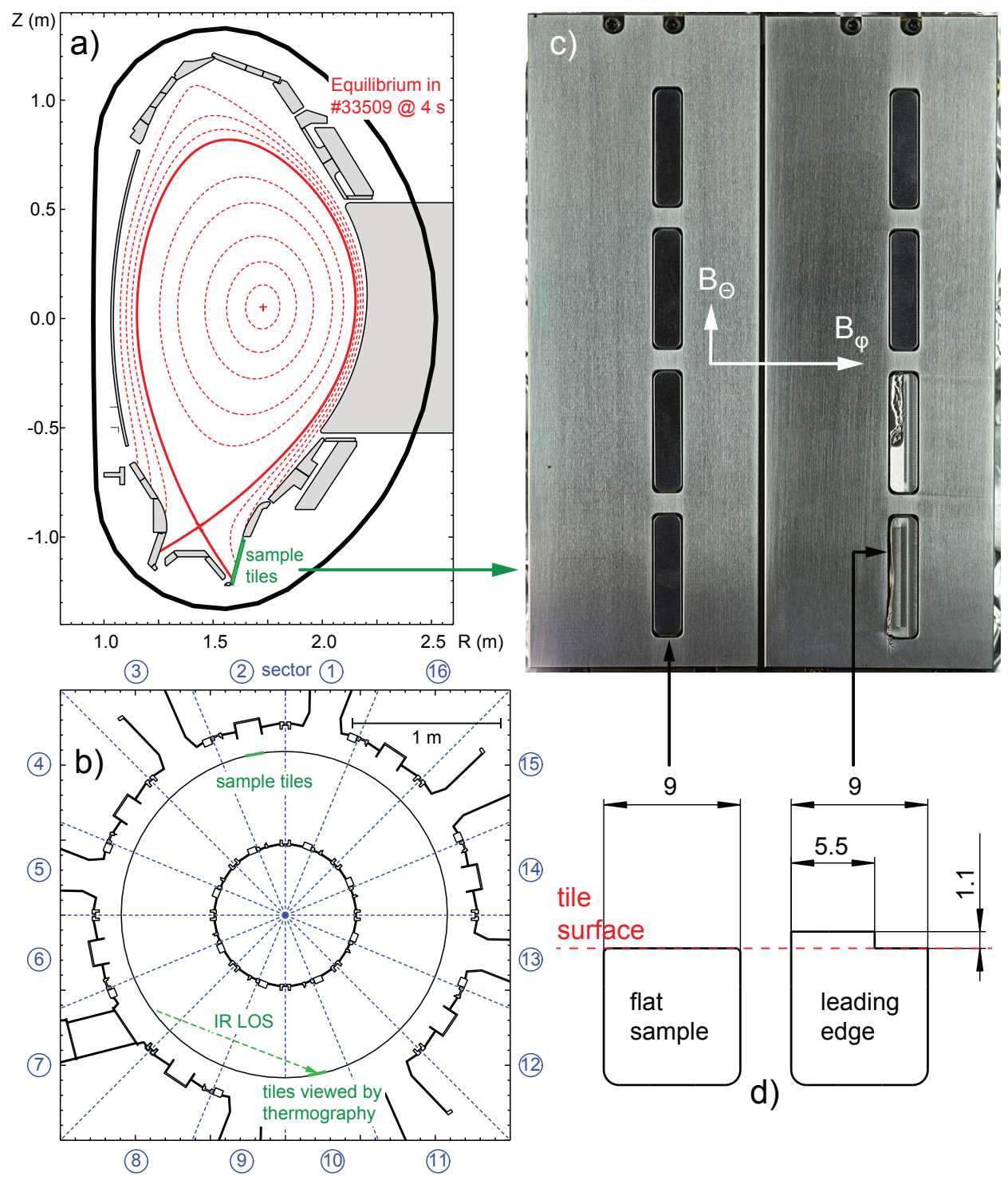

Figure 1. Details of the experimental set-up. (a) Cross-section of the AUG first wall components with the flux surface contours of magnetic equilibrium for the exposure of the leading edge sample in discharge \#33509. (b) Top view of the AUG vessel with the positions of the manipulator sample tiles and the thermography tiles. (c) View from plasma side to the surface of the two manipulator sample tiles with embedded bulk W samples post exposure. (d) Cross-sections of the flat bulk W reference samples and of the $\mathrm{W}$ melt sample with the leading edge facing the parallel divertor plasma flux.

from the heat flux derived for discharge \#33511 (in which in fact the upper sloped sample was exposed) with an appropriate downward shift of the profile anchored to the measured position of the outer strike point (OSP) in pulse \#33504.

Since the heat flux incident on the misaligned component was not directly measured, the heat flux distribution along the leading edge was evaluated by combining the optical approximation (in which particles are assumed to follow magnetic fields lines and any gyro-orbit motion is ignored) with the parallel heat flux deduced from outer target IR 
observations at a toroidal location distant from the sample [5], see also Fig.1b. Two representations of this heat flux, equivalent in terms of total energy delivered, have been employed in the simulations; (i) an analytic fit of the spatio-temporal dependence of the ELM heat flux after coherent averaging that is repeated in the modelling for the duration at which the sample is loaded at the average ELM frequency of $67 \mathrm{~Hz}$ (ii) the raw heat flux profile and time-series after corrections have been applied to subtract IR artifacts due to reflections and bremsstrahlung, for details see Ref.[5]. This latter option is the only suitable way to account for spatio-temporal variations in the heat flux without having to introduce arbitrary stochastic fluctuations in the coherent ELM waveform. In Fig.2, the spatial and temporal profile of the coherent structure as well as several snapshots from the corrected raw data are displayed. The inter-ELM heat flux, derived from Langmuir probes (agreeing within 10\% with IR data) [5], is superposed on the coherent ELM. It is described by a spatial fit of the same nature as the ELM heat flux, with a peak value of $\sim 50 \mathrm{MW} / \mathrm{m}^{2}$, and is assumed to be constant in time.

The measurements of the time-dependence of the total current flowing through the lamella to the grounded vessel (sampled at $200 \mathrm{kHz}$ ) constitute an important constraint for modelling. After the subtraction of the current flowing through the flush mounted reference sample (originating mostly from thermoelectric currents flowing between the two divertor targets), the remaining current has been attributed to the replacement current triggered by thermionic emission from the heated surface [5].
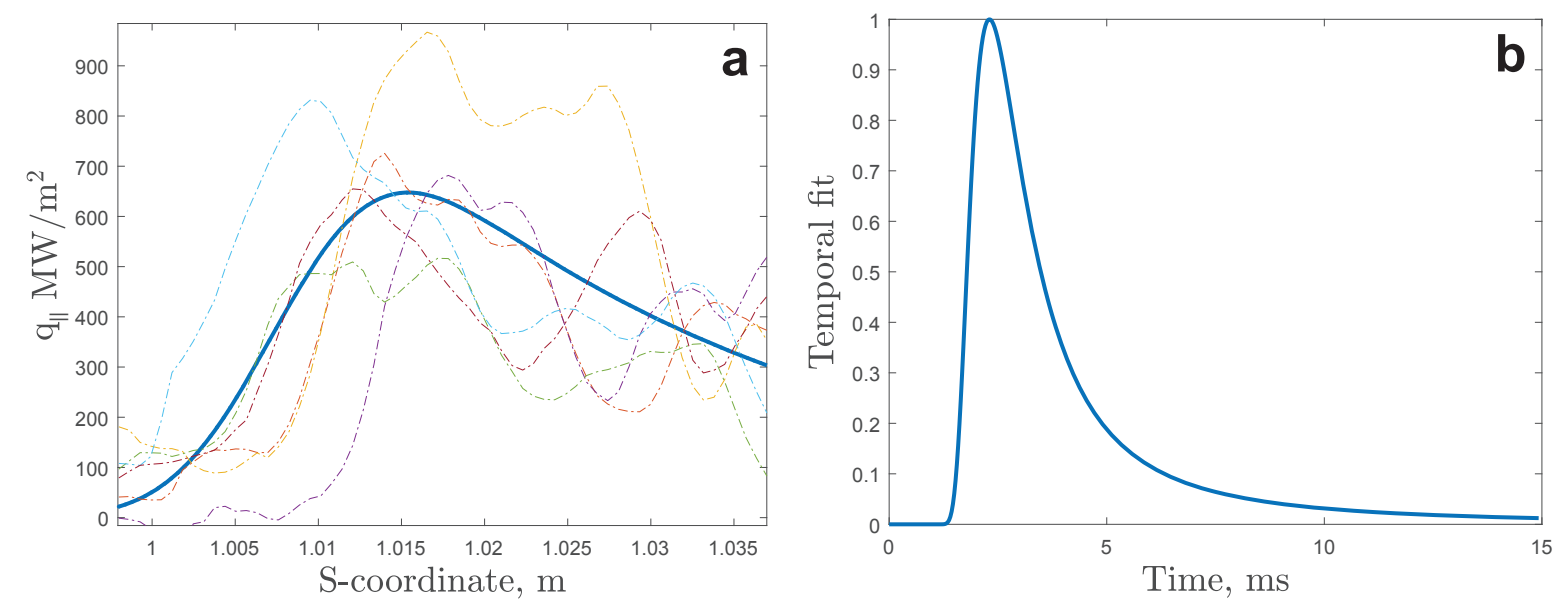

Figure 2. Spatial and temporal dependence of the ELM heat flux used as input to the MEMOS 3D modelling. (a) Solid line: Spatial distribution at the temporal maximum of the analytic fit to the coherent ELM. Dashed lines: Snapshots of the peak heat flux values extracted from the corrected raw data. Notice the strong fluctuations of the corrected raw data around the coherent averaged waveform, the relative value of the standard deviation at the peak of the coherent ELM is $\sim 40 \%$. (b) Temporal profile of the fitted coherent ELM within an ELM period time window (the choice of $t=0$ is arbitrary). The spatio-temporal coherent averaged heat flux is obtained by multiplying the two profiles. 


\section{Implementation in the MEMOS $3 \mathrm{D}$ code}

In this section, we shall briefly describe the status of the MEMOS 3D code. In particular, we outline some recent updates relevant for the simulations reported here.

The temperature dependence of the $W$ thermophysical properties, entering the heat transfer and hydrodynamic modules, have been updated according to the analytical formulas recommended in a recent survey [9]. The vapour shielding (but not vapour cooling) has been switched-off since the surface temperatures attained in the AUG experiments are relatively modest, with values typically not exceeding $4000 \mathrm{~K}$.

The boron-nitride (BN) substrate which provides the electrical isolation of the W sample, being an efficient heat sink, has been introduced due to the necessity to closely mimic the heat pathways present in the experiments. For the high purity hot isostatically pressed BN employed in AUG, the room temperature thermal conductivity and electrical resistivity are $47 \mathrm{~W} /(\mathrm{mK})$ and $3 \times 10^{21} \Omega \mathrm{cm}$, respectively. The temperature dependence of the heat capacity and thermal conductivity of the specific BN grade have been considered and a perfect thermal contact has been assumed at the W-BN boundary. The simulations revealed that for the short leading edge exposure simulated (the OSP is stable only for $0.5 \mathrm{~s}$ ), heat diffusion to the BN substrate becomes significant only after the OSP is shifted away from the lamella. Hence, below we report only on results for a thermally insulated W lamella.

The treatment of thermionic emission in MEMOS 3D has been significantly updated. The nominal or unimpeded thermionic current density follows the RichardsonDushman formula $j_{\mathrm{th}}^{\mathrm{RD}}\left(T_{\mathrm{s}}\right)=A_{\mathrm{eff}} T_{\mathrm{s}}^{2} \exp \left(-W_{\mathrm{f}} / k T_{\mathrm{s}}\right)$ where the values $W_{\mathrm{f}}=4.55 \mathrm{eV}$ and $A_{\text {eff }} \simeq 60 \mathrm{Acm}^{-2} \mathrm{~K}^{-2}$ have been employed for $\mathrm{W}$, see Ref.[7] for further justification. Current suppression due to space-charge effects has been quantified with the aid of systematic particle-in-cell simulations that had been carried out for the AUG inter- and intra-ELM plasma parameters with the 2D3V SPICE2 code $[7,8]$. The PIC results for the space-charge limited current and the virtual cathode magnitude agreed exceptionally well with the predictions of an analytical theory for finite surface temperatures and cold ions [10], revealing only small systematic deviations [8]. The PIC limited current density was observed to closely follow a Child-Langmuir law that reads as $j_{\text {th }}^{\lim }=0.43 e n_{\mathrm{e}} v_{\mathrm{Te}}$, where $n_{\mathrm{e}}$ and $v_{\mathrm{Te}}$ are the plasma density and electron thermal velocity, respectively [8]. This relation has been transformed to an expression connecting the limited current with the parallel heat flux under the additional assumptions: (i) $T_{\mathrm{i}}=T_{\mathrm{e}}$ for the ion temperature, (ii) $c_{\mathrm{s}}=\sqrt{\left(T_{\mathrm{e}}+T_{\mathrm{i}}\right) / m_{\mathrm{i}}}$ for the sound speed, (iii) $\gamma=7$ for the sheath heat transmission coefficient, (iv) $n_{\mathrm{e}}=$ const. implying that the heat flux spatio-temporal variations have been attributed to temperature variations. These assumptions lead to the simple expression $j_{\text {th }}^{\lim }\left(q_{\|}\right)=1.51 \times 10^{-4} q_{\|}^{1 / 3}$, where $q_{\|}$denotes the parallel heat flux. Supplementing the Richardson-Dushman formula with the above limiting value, we simply have $j_{\mathrm{th}}\left(T_{\mathrm{s}}, q_{\|}\right)=\min \left\{j_{\mathrm{th}}^{\mathrm{RD}}\left(T_{\mathrm{s}}\right), j_{\mathrm{th}}^{\lim }\left(q_{\|}\right)\right\}$. Equivalently, denoting the solution 
of $j_{\mathrm{th}}^{\mathrm{RD}}\left(T_{\mathrm{s}}\right)=j_{\mathrm{th}}^{\lim }\left(q_{\|}\right)$with $T_{\mathrm{s}}^{\lim }\left(q_{\|}\right)$, we end up with

$$
j_{\mathrm{th}}\left(T_{\mathrm{s}}, q_{\|}\right)= \begin{cases}A_{\mathrm{eff}} T_{\mathrm{s}}^{2} \exp \left(-\frac{W_{\mathrm{f}}}{k T_{\mathrm{s}}}\right) & T_{\mathrm{s}} \leq T_{\mathrm{s}}^{\lim }\left(q_{\|}\right), \\ 1.51 \times 10^{-4} q_{\|}^{1 / 3} & T_{\mathrm{s}} \geq T_{\mathrm{s}}^{\lim }\left(q_{\|}\right) .\end{cases}
$$

The obvious advantage of this treatment is that it enables us to directly reflect the effect of the spatio-temporal heat flux variations in the thermionic current, rather than introducing suppression factors for a restricted combination of plasma parameters. Note that thermionic emission contributes to cooling with an energy release of $2 k T_{\mathrm{s}}+W_{\mathrm{f}}$ per emitted electron $[7,11]$. To allow for a comparison with the total current versus time measurements, Eq.(1) is determined for every surface element at each time instant and integrated over the plasma-facing sample surface, i.e. $I_{\mathrm{th}}(t)=\int j_{\mathrm{th}}\left[T_{\mathrm{s}}(S, t), q_{\|}(S, t)\right] d S$. The former treatment of space-charge effects in MEMOS 3D $[6,12]$ followed the analytical theory of Ref.[10] in the heuristic limit of cold emitted electrons and did not consider the influence of the spatio-temporal variations of the incident plasma on the thermionic suppression factor. In addition, outdated thermionic constants were employed and the work function was erroneously not included in thermionic cooling.

The physics of the replacement current density that is responsible for the $\boldsymbol{J} \times \boldsymbol{B}$ force driving the melt motion [1] has been revisited. This current flows through the bulk of the lamella in order to replenish the thermionic electrons emitted at the plasmafacing surface. The Lorentz force acting on each volume element of the melt layer is determined by the magnetic field $\boldsymbol{B}$ and the local replacement current density $\boldsymbol{J}$, with two contributions at each spatial direction. The dominant force contribution is along the poloidal direction; it is the source of the experimentally observed poloidal melt motion and stems from the radial current density component $J_{\text {eff }}$ and toroidal magnetic field. The computation of the replacement current at each point of the melt layer is a non-trivial issue, even when considering the steady state problem in the absence of magnetic fields. The boundary value problem consists of the current continuity equation $\nabla \cdot \boldsymbol{J}=0$, the irrotational constraint for the electrostatic field combined with Ohm's law $\left(\rho_{\mathrm{el}}\right.$ is the dc resistivity) $\rho_{\mathrm{el}}(\nabla \times \boldsymbol{J})+\left(\nabla \rho_{\mathrm{el}}\right) \times \boldsymbol{J}=0$ and is complemented by appropriate boundary conditions that include thermionic emission as a current sink. Numerical solutions have been obtained with the finite element method (COMSOL Multiphysics) and revealed details of the current paths, the relative magnitude and spatial dependence of the different $\boldsymbol{J} \times \boldsymbol{B}$ components acting on the melt layer. For brevity these results are not, however, presented here. For simplicity, only the upper limit of the dominant component has been considered; it is assumed that at each fluid element $J_{\text {eff }}$ is equal to the current density emitted from the surface element directly above in the direction perpendicular to the plasma-facing edge.

\section{Modelling results}

In \#33504, the OSP was shifted down to its stationary position on the $\mathrm{W}$ sample $\left(s_{0}=1.008 \mathrm{~m}\right)$ at $t=3.3 \mathrm{~s}$ and was maintained there until $t=3.8 \mathrm{~s}$, when it was shifted 
upwards off the lamella. Fig.3 compares the measured and the computed currents for MEMOS 3D runs with coherent averaged (a) and corrected raw (b) heat fluxes, from the start of the OSP sweep to the sample $(t=3.0 \mathrm{~s})$. Unfortunately, the saturation level of the measurement system is reached during the ELM spikes within the $3.65-3.95 \mathrm{~s}$ span and the recorded current is truncated, hindering comparison with simulations in this interval. The initial phase of the experimental data (in blue) reveals peaks nearly from the start of the OSP sweep to the sample, which cannot be reproduced by modelling (in red) even by imposing large uncertainty margins in the heat flux magnitude and the OSP timing. In particular, surface temperature excursions during ELMs are $\sim 1000 \mathrm{~K}$ and to attain temperatures close to the $\mathrm{W}$ melting point, where thermionic emission becomes significant, a substantial increase of the bulk lamella temperature is required. In the coherent ELM case, $\sim 0.3 \mathrm{~s}$ from the start of the OSP sweep are needed for the inter-ELM surface temperature to reach values above $2000 \mathrm{~K}$. Thus, the highest current due to thermionic emission possible in the initial phase is that at $T_{\mathrm{s}} \sim 3000 \mathrm{~K}$ (reached at $3.3 \mathrm{~s}$ ), yielding $5 \mathrm{~A}$. As clearly illustrated in the figure, this value is exceeded in the current measurements much earlier than $3.3 \mathrm{~s}$. Such a significant discrepancy indicates that the measured current cannot be solely attributed to thermionic emission and that a current source of different nature should be present in the signal. In fact, in the latest AUG experiments, where current measurements were accompanied by simultaneous surface temperature measurements [13], significant current peaks were already detected for temperatures below $1500-2000 \mathrm{~K}$. The origin of this additional current source is presently not understood.
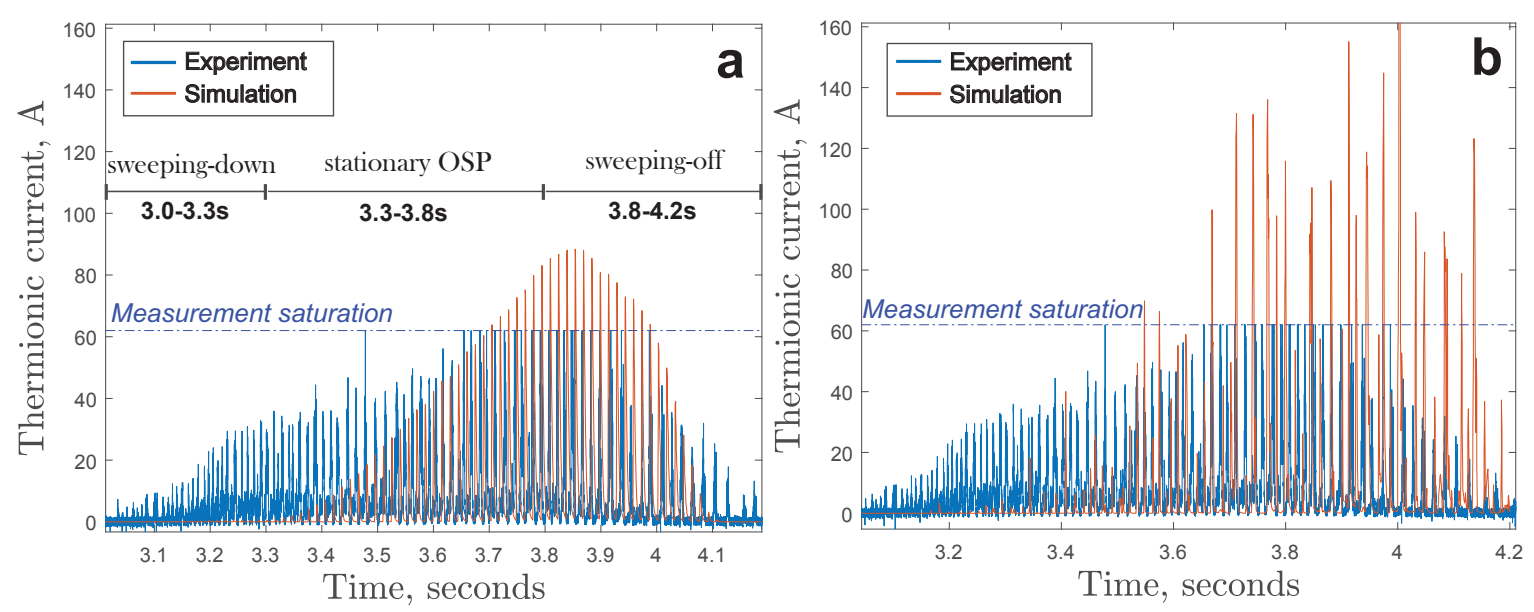

Figure 3. Comparison of the temporal dependence of the integrated thermionic current computed from MEMOS simulations (red) with the experimental current measurements (blue), for the leading edge exposure in shot \#33504 (the OSP timings are also indicated). Simulations employing the (a) coherent averaged heat flux and (b) corrected raw heat flux data; the initial deviations indicate that there is an additional current source other than thermionic emission, the subsequent deviations stem from the heat flux uncertainties and the overcoming of the upper measurement threshold. 

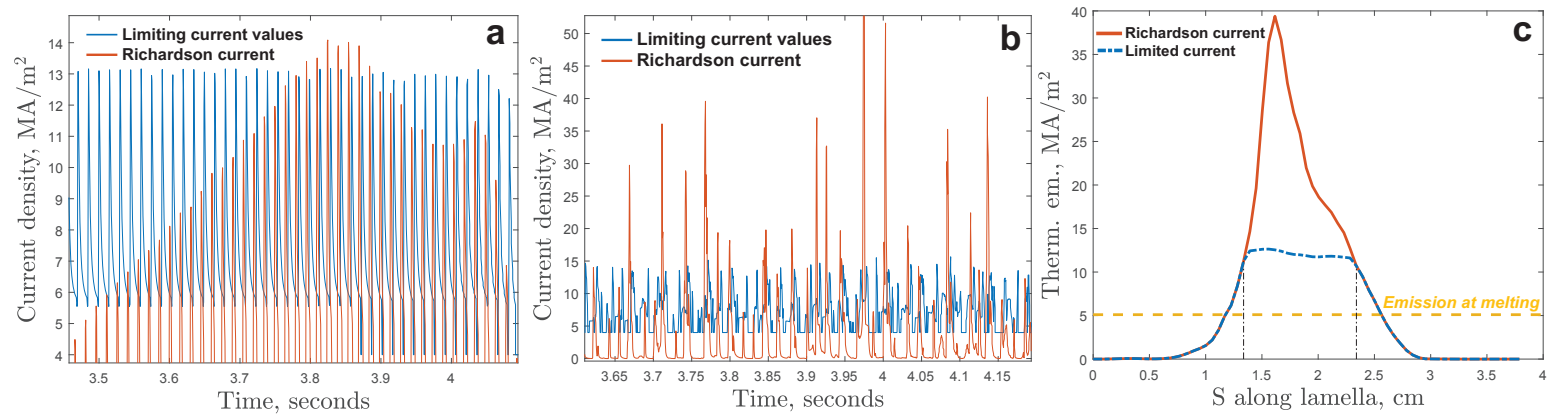

Figure 4. The unimpeded thermionic current density $j_{\mathrm{th}}^{\mathrm{RD}}\left(T_{\mathrm{s}}\right)$ according to the Richardson-Dushman formula (red) for the point of maximum temperature on the plasma-facing edge and the heat-flux dependent limiting current density value $j_{\mathrm{th}}^{\lim }\left(q_{\|}\right)$. Simulations with (a) coherent averaged and (b) corrected raw heat flux data; the spacecharge limited regime is reached for a restricted time in the former case and for a more extended overall duration in the latter case. (c) Snapshot of a large event from the corrected raw heat flux runs quantifying the relative contributions of the unimpeded current (blue) and the limited current (red), the vertical lines indicate entry to the space-charge limited regime.

The computed thermionic current reproduces the temporal behaviour and lies within an order of magnitude of the measured current, but none of the simulations approach the measurements quantitatively. This is not caused solely by the unknown contribution of the additional current source, but also because the coherent flux averages out some important characteristics; it repeatedly strikes the same position with identical magnitude so that the emerging thermionic current envelope is monotonically increasing (due to the base temperature increase on which nearly constant ELM temperature excursions are superimposed). On the other hand, as clearly illustrated in Fig.2, the individual ELMs can have significant variations not only in the heat flux profile and the position of its maximum but also in the energy delivered. These characteristics allow the reproduction of variations present in the measured signal, namely occasional "violent" current bursts followed by "quiescent" periods. However, a one-to-one correspondence is unfeasible since the heat flux data are from another discharge and are measured at a toroidal location very far from the W sample (ELMs are inherently toroidally asymmetric). These differences are also reflected in the spatio-temporal behaviour of the surface temperature and melt depth, see below.

To attribute the measured total current to thermionic emission, a comparison with the Richardson current is usually performed using oversimplified assumptions for the emitting area and the surface temperature, typically considered equal to the molten region and the melting point or above, respectively $[3,12]$. With the simulation results we are in position to identify the contributions to the total current from different regions of the wetted area. Fig.4 shows the limiting current values $j_{\mathrm{th}}^{\lim }\left(q_{\|}\right)$(in blue) and the unimpeded thermionic current $j_{\text {th }}^{\mathrm{RD}}\left(T_{\mathrm{s}}\right)$ according to the Richardson-Dushman formula (in red) for the coherent (a) and corrected raw heat fluxes (b). Note that, 
owing to cooling by thermionic emission, for the comparison to be meaningful, $j_{\mathrm{th}}^{\mathrm{RD}}\left(T_{\mathrm{s}}\right)$ was calculated for the surface temperature profiles obtained from runs with limited thermionic emission following Eq.(1). Fig.4a shows that for the coherent flux, even though from $3.7 \mathrm{~s}$ and onwards part of wetted area is molten, the limited regime is only entered in a few instances. In contrast, Fig. $4 \mathrm{~b}$ reveals that for the corrected raw flux a significant number of (but not all) ELMs bring thermionic emission into the limited regime (with large, factor of 2-3 differences between the unimpeded and limiting values). In a spatial snapshot of one of the larger events, as in Fig.4c, the total current is observed to comprise of two comparable contributions; from the area with temperature exceeding the critical value for a given heat flux and thus emitting at the limiting value (between the vertical lines, under the red curve) and from the remaining area emitting the unimpeded Richardson current (wings, left and right of the vertical lines).
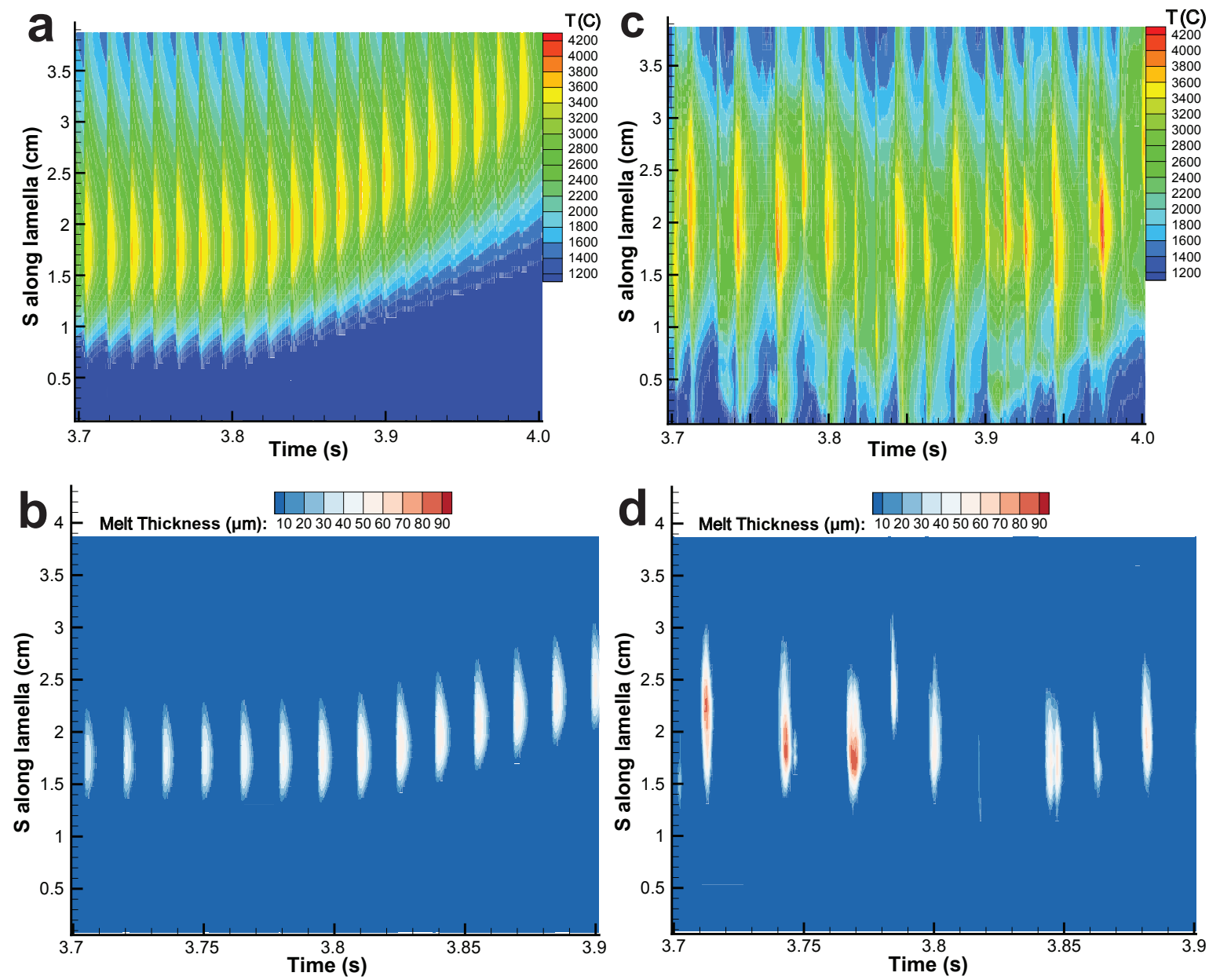

Figure 5. Temporal dependence of the poloidal profile of the surface temperature (upper row) and the thickness of the melt pool (lower row) at the uppermost edge of the exposed surface. Simulations with $(\mathrm{a}, \mathrm{b})$ coherent averaged and $(\mathrm{c}, \mathrm{d})$ corrected raw heat flux data; the coherent heat flux leads to a progressive increase of the melt pool dimensions during each ELM in contrast to the corrected raw heat flux. 
The necessity to include the stochastic character of the spatio-temporal behaviour of the ELM heat fluxes can be further highlighted by comparing the basic figures-ofmerit of the molten area, namely the poloidal extension and the melt depth. The bottom panel of Fig. 5 illustrates the melt pool formed during each ELM for coherent averaged (b) and corrected raw heat fluxes (d), starting from $3.7 \mathrm{~s}$, when the base temperature first approaches $\sim 3000 \mathrm{~K}$ and melting becomes significant. The poloidal melt extent and melt depth range respectively within $0.9-1.1 \mathrm{~cm}, 40-55 \mu \mathrm{m}$ for the coherent and $0.8-1.7 \mathrm{~cm}, 40-90 \mu \mathrm{m}$ for the corrected raw ELM heat flux. The experiment yields $\sim 1.5 \mathrm{~cm}$ for the poloidal extent as deduced from the final erosion profile of the lamella and $\sim 100 \mu \mathrm{m}$ for the pool depth as deduced from SEM images of the re-solidified melt [5]. The displaced melt volume (the "build-up") is $0.4 \mathrm{~mm}^{3}$ and $0.7 \mathrm{~mm}^{3}$ for the coherent and corrected raw ELMs, respectively. These values are to be compared with the experimentally observed displaced molten volume, $\sim 7 \mathrm{~mm}^{3}$, obtained from the final erosion profile [5]. Here, we should emphasize that surface analysis was carried out postmortem and documented the cumulative effect of all discharges - \#33504 (simulated here), \#33508, \#33509. It is also worth noting that the last exposure was appreciably longer: the OSP was held on the sample for $1.1 \mathrm{~s}$ in contrast to the $0.5 \mathrm{~s}$ simulated here. Since melt build-up does not scale linearly with the exposure time due to the base temperature evolution, resulting in more efficient melting (deeper melt pools, stronger thermionic emission) at later stages, the main contribution to the experimental value is due to the last longest exposure and thus there is no disagreement with the simulations. Indeed, longer runs, where the stable OSP duration was simply extended, lend full support to such interpretation.

\section{Summary}

Melt motion simulations of recent AUG experiments on $\mathrm{W}$ melting under transient heat loads have been performed with MEMOS 3D. It has been concluded that the measured current cannot be solely induced by thermionic emission, an additional current source must be present whose origin is presently not understood. The possible contribution of the additional current density to macroscopic erosion will mainly depend on its ratio over the thermionically triggered replacement current density inside the molten parts of the $\mathrm{W}$ lamella. The quantification of this contribution is one of the main objectives of new experiments planned on AUG to follow-up on those discussed here and in Ref.[5]. The total current measurements as well as the updated description of the space-charge limitation in MEMOS also allowed for a detailed investigation of the role of thermionic emission. The scaling of limiting current with the heat flux as given by Eq.(1) results in an intimate coupling with the spatio-temporal details of the heat flux. An aperiodic heat flux pattern, including temporarily sharper ELMs, implies inhibited conduction and thus higher surface temperatures leading to a more efficient passage of the thermionic current into the limited regime. Hence, the whole interplay between the limited current and melt motion is different from the case of a periodic coherent heat load. Variations in 
the spatial heat flux density profile have a dual effect as well; they prevent overheating of the same spot by distributing the heat in different location at each ELM but also allow for higher local energy density. The latter again implies more efficient switching to the limited regime.

\section{Acknowledgments}

This work has been carried out within the framework of the EUROfusion Consortium and has received funding from the Euratom research and training programme 2014-2018 under grant agreement No 633053. Work performed under WP MST1. The views and opinions expressed herein do not necessarily reflect those of the ITER Organization or of the European Commission. ITER is the nuclear facility INB 174.

[1] Pitts R A, Bardin S, Bazylev B et al. 2017 Physics conclusions in support of ITER W divertor monoblock shaping Nucl. Mat. Energy http://dx.doi.org/10.1016/j.nme.2017.03.005

[2] Pitts R A, Carpentier S, Escourbiac F et al. 2013 J. Nucl. Mater. 438 S48

[3] Sergienko G, Bazylev B, Hirai T et al. 2007 Phys. Scr. T128 81

[4] Bazylev B, Janeschitz G, Landman I et al. 2009 J. Nucl. Mater. 390-391 810

[5] Krieger K, Coenen J W, Matthews G F et al. 2017 Nucl. Fusion (submitted)

[6] Coenen J W, Arnoux G, Bazylev B, Matthews G F et al. 2015 Nucl. Fusion 55023010

[7] Komm M, Ratynskaia S, Tolias P et al. 2017 Plasma Phys. Control. Fusion 59094002

[8] Komm M, Tolias P, Ratynskaia S et al. 2017 Phys. Scr. (these proceedings)

[9] Tolias P 2017 arXiv:1703.06302v4

[10] Takamura S, Ohno N, Ye M Y and Kuwabara T 2004 Contrib. Plasma Phys. 44126

[11] Herring C, and Nichols M H 1949 Rev. Mod. Phys. 21185

[12] Coenen J W, Philipps V, Brezinsek S et al. 2011 Nucl. Fusion 51083008

[13] Krieger K, Sieglin B, Balden M et al. 2017 Phys. Scr. (these proceedings) 